\title{
PSICOLOGÍA ORGANIZACIONAL: APLICACIÓN EN LA VIDA PROFESIONAL
}

ORGANIZATIONAL PSYCHOLOGY: APPLICATION IN THE PROFESSIONAL LIFE

Rafael Elizade ${ }^{1}$, Marybel Mollo F., Jeny Flores V.

Universidad Nacional Mayor de San Marcos, Perú.

(RECIBIDO El 21/07/2010, ACEPTADO EL 5/10/2010

\section{RESUMEN}

El presente artículo pretende brindar luces acerca de la importancia de la Psicología Organizacional en el ámbito laboral y ayudarnos a comprender cómo ésta puede resolver conflictos en la organización complementándose con diferentes ámbitos profesionales.

Los autores, no psicólogos, que cursan el último semestre de la Maestría con mención en Psicología Organizacional de la UNMSM, expondrán la utilidad que la Psicología Organizacional les brinda en su quehacer profesional aplicado en las organizaciones donde ellos se desempeñan.

Palabras clave: Psicología organizacional, inteligencia emocional, procesos del trabajo, evaluación de desempeño laboral, informática, educación.

\begin{abstract}
This article attempts to provide insights into the importance of organizational psychology in the workplace and also how it can help resolve conflicts in the organization and supplemented with different professional fields

The authors, not psychologists, who enrolled in the final half of the Masters with a specialization in Organizational Psychology at UNMSM, expose the utility gives Organizational Psychology in their professional duties applied in the organization where they perform.
\end{abstract}

Keywords: Organizational psychology, emotional intelligence, work processes, evaluation of job performance, computing, education. 


\section{INTRODUCCIÓN}

No cabe duda que con el desarrollo económico del país en los últimos años, se vienen presentando innumerables iniciativas de emprendedores que desean aprovechar esta ola de crecimiento financiero, estructurándose así nuevas organizaciones encargadas de brindar productos y servicios para el Perú y el extranjero.

Debemos comprender que las sociedades entonces no solo se componen de individuos con nombre y apellido, sino también de organizaciones formal o informalmente conformadas, grupos sociales, entidades de ayuda, etcétera, las cuales van tomando importancia en su rol social y que según Darío Rodríguez manejan dimensiones como la cultura organizacional, el clima organizacional, los procesos y el tiempo, mediante las cuales es posible determinar un diagnóstico de la organización de manera similar a como un psicólogo clínico lo haría con su paciente.

Es así, que de una u otra forma y al desarrollar la vida profesional y laboral, el individuo va tomando atención a circunstancias que le hacen cuestionar el porqué, el cuándo y el cómo de conceptos tales como el ambiente de trabajo, el estrés, la desmotivación, el compromiso, la desidia, el máximo rendimiento, el reconocimiento, la empatía con la institución y todos aquellos factores que constituyen su relación con la organización. Temas que poco a poco van generando una necesidad de respuesta y que si bien podemos tener claro nuestro quehacer profesional, es menester conocer éste complemento teórico que responderá a estas interrogantes: la Psicología Organizacional.

Fue este interés que actuó como estímulo en los autores, llevándolos a iniciar estudios en Psicología Organizacional buscando tal vez respuesta a estas preguntas pues ellos consideran que la Psicología Organizacional puede complementarse con otras líneas de investigación en base a un trabajo interdisciplinario y por lo tanto también debe de ser acogida en la formación profesional.

\section{LA PSICOLOGÍA ORGANIZACIONAL EN EQUIPOS DE TRABAJO DE SISTEMAS INFORMÁTICOS}

Desde el ámbito informático, el Lic. Rafael Elizalde considera que la demanda de profesionales informáticos que, además de conocimientos técnicos, deban poseer habilidades emocionales es cada día mayor. Estas habilidades que comprenden tener facultades interpersonales para el trabajo en equipo o para hablar en público, capacidad de liderazgo o de adaptación al cambio como componentes de su perfil profesional son importantes en cualquier trabajo hoy en día.

Para J. M. Peiró (1996), quién hace una taxonomía del ambiente laboral, los Procesos del Trabajo son una dimensión del ambiente de trabajo, donde se encuentran los aspectos temporales y la secuencialidad del trabajo (duración de la jornada laboral, ritmo y cadencia del proceso productivo, variaciones en el flujo de trabajo, presiones y fechas tope para su finalización, el nivel de exigencias de proceso (estándares de calidad, picos en la producción), la amplitud en la supervisión, la sobrecarga cualitativa y cuantitativa (tanto física, como psicológica y emocional), la postura de trabajo o la secuencialidad de movimientos, así como la interdependencia funcional con otras áreas y el flujo de información, productos y procesos de trabajo. 
Bajo la experiencia profesional se ha logrado identificar circunstancias laborales en donde la presión de estos procesos del trabajo producen estadíos de tensión y fricción entre el personal del área, que merman el ambiente laboral y la productividad.

Por otro lado, en el curso de los últimos años, el cociente emocional (CE) se viene aceptando ampliamente como una expresión abreviada del equivalente en inteligencia emocional al cociente de inteligencia (CI). Las investigaciones recientes rescatan que un profesional con un alto $\mathrm{CE}$ es una persona que percibe más hábil, fácil y rápidamente que los demás los conflictos en gestación, los puntos vulnerables de los equipos y organizaciones, las distancias que deben salvarse o los vacíos que se deben llenar.

Autores como Daniel Goleman (1995) y Robert Cooper (1997) plantean la aplicación de la Inteligencia Emocional al liderazgo y a las organizaciones. Para Goleman, las competencias emocionales son: autoconciencia, autodirección, aptitudes sociales, y relaciones de dirección, mientras que para Cooper su modelo de cociente emocional se basa en cuatro pilares: alquimia emocional, conocimiento emocional, profundidad emocional y aptitud emocional.

Por su parte, para Reuven Bar-On (2000), según su modelo de Inteligencia Emocional, existen capacidades facilitadoras, como el optimismo, la alegría, la independencia emocional, la autorrealización y la responsabilidad social. Elementos que ayudarán a la convivencia dentro de la organización. Así por ejemplo, la asertividad se relaciona con la autoseguridad y la resolución de problemas dependerá del optimismo, de la flexibilidad y el manejo de la presión.

\section{LA PSICOLOGÍA ORGANIZACIONAL EN LA GESTIÓN ADMINISTRATIVA}

Desde la Psicología Organizacional, la bachiller en Lingüística Marybel Mollo Flores busca respuestas para solucionar un problema de Recursos Humanos que tiene el instituto de idiomas en el cual trabaja: la evaluación de desempeño laboral.

Para Martha Alles (2006), la función básica del trabajo de una persona es el de producir resultados sin importar cuál sea su posición o puesto de trabajo. Siempre interesa que un empleado deje algún tipo de utilidad. Para que estos resultados se concreten, la persona debe conocer "qué se espera" y a su vez recibir feedback, es decir, que alguien le diga cómo lo está haciendo y ser recompensado por ello.

Por otra parte, la definición por competencias se relaciona con la mejor manera de lograr objetivos diseñados a su vez en función de esas competencias que llevarían al éxito a la organización.

Los enfoques para relacionar competencia con desempeño son varios. Por un lado, el management de la organización fija los objetivos que caen en cascada a toda la empresa o institución. Estos objetivos tienen directa relación con los objetivos organizacionales. Cuando a los empleados se les fijan los objetivos, ellos son parte de esa "cascada de objetivos organizacionales". Para Mariano Bernárdez (2006), las competencias y comportamientos son medios para mejorar la performance si y solo si producen mejores resultados con menor esfuerzo o reducen el coste de lograr esos resultados. 
Desde la perspectiva del empleado, esta fijación de objetivos le permite formar parte de la empresa, lo ayuda a vincular su quehacer diario con los objetivos organizacionales y con los resultados. En este sentido, Robert Mathis (2000) plantea la evaluación del desempeño relacionando la estrategia global de los negocios con los objetivos individuales del empleado.

Podemos considerar entonces, como los objetivos más importantes de la evaluación del desempeño:

a. El desarrollo personal y profesional de los colaboradores.

b. La mejora permanente de los resultados de la organización.

c. El aprovechamiento adecuado de los recursos humanos.

Según Martha Alles (2006), las evaluaciones deben ejecutarse en base al perfil del puesto de trabajo y éstas son necesarias porque permiten:

a. Tomar decisiones de promociones y remuneración.

b. Reunir y revisar las evaluaciones de los jefes y subordinados sobre el comportamiento del empleado en relación al trabajo. Recordemos a Edgar Schein cuando explica que un trabajador "necesita saber cómo se está realizando su trabajo", el grado de satisfacción que sus empleadores tienen en relación con la tarea realizada.

c. La mayoría de las personas necesitan y esperan retroalimentación; a partir de conocer cómo hacen la tarea, pueden saber si pueden modificar su comportamiento.

\section{LA PSICOLOGÍA ORGANIZACIONAL EN LA ORGANIZACIÓN EDUCATIVA ESTATAL}

Por su parte la Lic. en Pedagogía Jeny Flores Vivas, enfoca la Psicología Organizacional como herramienta de gestión eficiente en las organizaciones educativas, sostiene además la necesidad de la intervención del psicólogo organizacional en el ámbito educativo por su facultad de organización y planificación.

Según José Luís Martorell (2009), los elementos a tener en cuenta en la organización eficiente de una entidad educativa para el talento humano son:

a. Selección. Misión de alta importancia en el área educativa, porque de esta selección depende en gran medida el futuro alcance de metas y objetivos, lo que involucra un despliegue conciente de capacidades frente al reto de selección, capacitación, actualización, orientación y evaluación permanente, al cúmulo de involucrados en el ámbito educativo en la ruta a seguir para alcanzar el objetivo planeado.

b. Formación y desarrollo. El asunto es bastante complejo en este punto, ya que involucra, por ejemplo, la devaluación del prestigio social de la carrera docente, la pobre calidad de la educación superior en el país, la proliferación de licencias y permisos de funcionamiento de la diversa gama de nacientes universidades privadas y centros e institutos de formación pedagógica que en los últimos años lamentablemente han demostrado no contar siquiera con un centro de aplicación de prácticas pr.profesionales y mucho menos solidez en la formación de los egresantes. 
c. Diagnóstico e intervención del ambiente laboral en el ámbito de la organización educativa. Según la PRELAC ${ }^{2}$ (2005) la suma de horas destinadas a trabajo docente, a trabajo doméstico y trabajos adicionales, arroja un cuadro de sobrecarga laboral en que cabe poco tiempo para el descanso y se convierte así en un factor de riesgo para la salud de los docentes, y ello debe considerarse en los planes preventivos. Al mismo tiempo, esto es un factor de amenaza para el mejoramiento del desempeño docente.

d. Dirección de entidades educativas. Al respecto el Ministerio de Educación como órgano representativo del Estado viene mostrando algunas luces de innovación y ánimo de reconocimiento al esfuerzo y dedicación de docentes del sector estatal poniendo en marcha la tan promocionada carrera pública magisterial cuya fortaleza radica en el reconocimiento económico a los docentes que superen satisfactoriamente las evaluaciones públicas de idoneidad profesional y desempeño académico.

\section{CONCLUSIONES}

1. Existen problemáticas organizacionales que escapan del alcance profesional para determinadas áreas. Dichas cuestiones encuentran respuesta en la Psicología Organizacional, como disciplina complementaria en el quehacer profesional.

2. La inteligencia emocional aporta habilidades emocionales que permiten al individuo superar problemas de interrelación, estrés, tolerancia al cambio, autorrealización y en general todos aquellos generados por los procesos del trabajo en la organización.

3. El desempeño como métrica de productividad del empleado requiere establecer las metas alcanzables y una constante retroalimentación de los resultados a través de mecanismos de auto evaluación como feedback laboral por parte de los empleados a través de formularios de fácil comprensión. Este método no puede quedar a entera responsabilidad de los mismos, sino luego se deben analizar en conjunto con su superior o jefe directo.

4. Hacer de la evaluación un ciclo continuo a través de la formación de comisiones de evaluación del desempeño con miembros permanentes y transitorios.

5. Por otro lado, los espacios insuficientes para que los docentes puedan preparar clases y materiales, falta de espacios para el descanso, falta de servicios básicos de saneamiento (servicios higiénicos, comedores, cocinas), y la distribución del material didáctico adecuado son las carencias en las que habitualmente se desempeña el docente y deben considerarse para adecuada estructuración y organización bajo el enfoque del psicólogo organizacional.

6. El Perú requiere líderes educadores con un adecuado enfoque en Psicología Organizacional que reflejen en sus proyectos educativos y conocimientos el afán de fortalecimiento y búsqueda de la calidad y excelencia educativa.

7. La Psicología Organizacional es sin duda un excelente complemento profesional en la conducción de organizaciones desde el aspecto de la cultura y el clima de organización.

2 PRELAC, Oficina Regional de Educación para América Latina y el Caribe. 


\section{REFERENCIAS BIBLIOGRÁFICAS}

1. Alles, Martha Alicia (2006). Desempeño por competencias: evaluación de $360^{\circ}$. 1a. ed. 3a. reimp. Buenos Aires: Granica.

2. Bernárdez L., Mariano (2006). Tecnología del desempeño humano. Indiana, EE.UU.

3. Chiavenato, Adalberto (1994). Administración de recursos humanos. Bogotá: Editorial Presencia.

4. Cooper, K. \& Sawaf A. (1997). La inteligencia emocional aplicada al liderazgo y a las organizaciones. New York.

5. García Álvarez, Ana I. (1999). El Feedback laboral en la gestión organizacional. Valencia, Promolibro.

6. Goleman, D. (1998). La inteligencia emocional en la empresa. Madrid.

7. Kolb, D.; Rubin, I.; Mcintyre, J. (1993). Psicología de las organizaciones: Problemas contemporáneos. México: Editorial Prentice Hall Hispanoamericana.

8. Martorell, José Luís; Prieto Arroyo, José Luis (2009). Fundamentos de psicología. Madrid. España.

9. Peiró, José María (1984). Psicología de la organización. 1. ${ }^{\text {ra }}$ ed., Madrid: Universidad Nacional de Educación a Distancia.

10. Peiró, José María y Prieto, Fernando (1996). Tratado de psicología del trabajo. Volumen 1. Madrid.

11. PRELAC, Oficina Regional de Educación para América Latina y el Caribe (2005). Condiciones de trabajo y salud docente. Santiago de Chile.

12. Robbins, Stephen. (2004). Comportamiento organizacional: Conceptos, controversias y aplicaciones. México: Editorial Prentice Hall Hispanoamericana.

13. Rodríguez, Darío (1999). Diagnóstico organizacional. 3. ${ }^{\text {a }}$ ed. Santiago, Chile.

14. Schein, Edgar. H. (1982). Psicología de la Organización. México: Prentice Hall.

15. Werther, William; Davis, Keith (1999). Administración de personal y recursos humanos. México: McGraw-Hill.

16. Zapata, Roberto (2004). Medición del impacto de la capacitación en la empresa (ROI). Apuntes del Curso impartido por Chilecapacita en octubre de 2004, Santiago de Chile. 